\title{
Developing International Clinical Placements: Enhancing Student Awareness
}

\author{
Sharon Elizabeth Metcalfe* \\ Western Carolina University, 28 Schenck Parkway, Suite 305, Asheville, North Carolina 28804, United States
}

Received Date: December 12, 2017 Accepted Date: December 21, 2017 Published Date: December 27, 2017

Citation: Sharon Elizabeth Metcalfe (2017). Developing International Clinical Placements: Enhancing Student Awareness. POJ Nurs Prac Res 1(4): 1-7.

*Corresponding Author: Sharon Elizabeth Metcalfe, Ed.D. MSN, RN, Associate Professor, Western Carolina University, 28 Schenck Parkway, Suite 305, Asheville, North Carolina 28804, United States, Tel: +828-776-7108;

Email: metcalfe@email.wcu.edu

\begin{abstract}
Throughout the globe, university schools of nursing have implemented foreign exchange excursions for nursing students to experience nursing care of a variety of patients from various cultures and ethnic backgrounds. There is a dearth of these clinical placements though that are focused upon developing student awareness of nursing placement leaders. This article describes the learning provided from clinical nursing mentors who guide the students in enhancing their awareness of cultural awareness, socialized and capitalistic healthcare, as well as the role of nursing leaders. This article's purpose is to showcase an international program that has been in existence for eleven years that has combined the educational and clinical focus of two international university schools of nursing and two children's hospitals that are located in a district in Great Britain, as well as the Southeastern United States. This program utilizes a special educational clinical placement method that has allowed both students from Great Britain and the Southeastern United States to select their clinical nursing mentor and specify the desired learning environment in both the hospital clinical and community environment. Both successes and challenges of the joint international clinical placement program are presented and the recommendations for other schools of nursing as well as clinical hospital agencies that desire to embark on developing a mutually satisfying clinical placement program.
\end{abstract}

Keywords: Clinical Placement, Clinical Nursing Mentor, Exchange Programs

\section{Introduction}

Internationally, the world population is becoming more culturally and ethnically diverse, and is impacting health professionals such as the nursing profession who is being asked to broaden their knowledge base to care for individuals from many multicultural backgrounds [1]. The Sullivan Commission Report on Diversity in the Healthcare Workforce (2014) highlighted the stagnation and imbalance in the makeup of the United States' diversity of all health professionals, including the nursing profession that had a diminished number of nurses that were from a wide spectrum of cultural and ethnic backgrounds [2]. In 2010, the Institute of Medicine made a recommendation in The Future of Nursing Report to diversify the student population of the health care professions in order to provide increasing nursing minority providers to meet the culturally competent needs of the growing multicultural populations of the United States [3].

Despite this mandate from the Institute of Medicine, the number of diverse graduates from the nursing programs in the United States continues to be low, as compared with the number of Caucasian graduates [4]. Current statistics report that considering racial/ethnic backgrounds, the registered nurse population is composed of $83 \%$ White/Caucasian, 6\% African American, 6\% Asian, 3\% Hispanic, 1\% American Indian/Alaskan Native, $1 \%$ Native Hawaiian/Pacific Islander, and $1 \%$ other nurses [5]. These statistics pale when compared with the increasing minority population that is multiplying within America. Recent statistics show that within the United States, the Census Bureau (2012) released estimates on the U.S. population's finding that racial and ethnic minorities for the first time made up more than half of all children born in the country, totaling $50.4 \%$ [6]. These high number of minority children that are being born in

Copyright: (C) 2017 Sharon Elizabeth Metcalfe. This is an open-access article distributed under the terms of the Creative Commons Attribution License, which permits unrestricted use, distribution, and reproduction in any medium, provided the original author and source are credited. 
the United States are not however being admitted to colleges and universities.

Preparing today's nursing students for the challenges that they will face with caring for a truly multicultural and ethnically diverse patient and client population requires a structured educational method that positively impacts the growth of nursing students' awareness. An answer to promote their knowledge base of diversity of care for these patients may be accomplished through experiencing international clinical placement experiences that focus upon the student being guided through carefully designed mentoring relationships. International clinical experience with specially selected nursing mentors for the experience has been found to facilitate the students' personal and professional growth and broaden their knowledge of global issues and understanding of different cultures [7]. International experiences are often developed to be service learning based with third world nations and provide experiences that are often unable to be obtained in the student's home university or college environment where the diversity of patients that are cared for in the rural health clinics does not exist [1].

The purpose of this article is to describe the development of a specially crafted and designed collaborative placement partnership program that has been in existence for eleven years between two universities and two hospitals that reside in both the Southeastern United States and a district in Great Britain. An additional purpose of this article is to facilitate the knowledge for faculty interested in developing programs of how to develop a similar program that focuses upon designing clinical placements with nursing mentors to guide the students' maturation and cultural awareness. These nursing mentors are located in a wide variety of nursing inpatient and outpatient community environments. These nurses enhance the development of the students that learn to care for patients that are extremely multicultural and diverse from the patients that are found in the hospitals and community at home for the visiting student. For both the visiting students from the United States, as well as Great Britain, the cultural awareness and care delivered by both socialized and capitalistic healthcare is presented and is an eye-opening experience.

\section{Review of Literature of International and Clinical Placements for Nursing Students}

The experience of traveling to another country that is uniquely different than one's own is often both enjoyable, as well as startling that tends to awaken the cultural awareness of the student regarding the inhabitants, as well as lifestyles, of the new environment. International experiences provide students with an opportunity to learn global health and nursing care issues, as well as the similarities and differences in care [8]. In a systematic review of 23 empirical articles regarding international student nursing exchange experiences that focused upon the use of clinical placement mentors that guided the development of the student, Kolbuck, Mitchell, Glick, and Grenier (2012) found that there were not any research or review studies that focused upon international clinical placement [9]. There was no evidence of international studies that focused upon the use of nursing mentors, and the studies were primarily focused upon the traditional use of foreign exchange service learning programs to third world countries.

Despite the dearth of studies and review articles of programs that have been based upon the development of international clinical placement with guidance of a nursing mentor, there have been a plethora of articles regarding the use of mentoring relationships and clinical placement. Dobrowolska et al. (2016) found in an extensive case study that surveyed eleven directors of university schools of nursing in eleven countries that there were no conclusive findings that showed the similarities in the role of clinical mentor and placement for the various students from the diverse nations [10].

Additional issues revealed that clinical placement mentors often had the primary role of caring for patients in the inpatient setting, and the role of assisting the student through a preceptor role was secondary. The authors recommended that each nation determine the role of the clinical placement mentor before the nations in the European Union could develop the role of the clinical placement mentor to guide undergraduate students to success of nursing care.

Ward and McComb (2017) conducted an in-depth literature review that sought to determine the role of the clinical preceptor and its importance to leading nursing students in the inpatient environment [11]. The review summarized that there was a wide variety of models that presented preceptorship and there was not one significant model. A recommendation for clinical preceptors was presented, although the review of literature was lacking regarding a clinical model of preceptorship. Clinical preceptorship roles were found through a literature review of eighteen studies that met the inclusion criteria for assessing the role of clinical mentoring that there existed the need for consistency and continuity, the potential for a variety of models of mentoring, and the variability/sustainability of the model [12]. Roxburgh (2014) found through a qualitative focus group approach with nursing students, mentors, and clinical managers that undergraduate students preferred having a clinical mentor that was devoted to them and this increased their faith in their ability to cope with the practice of learning and the educational demands of the nursing profession [13].

Despite the importance of clinical mentoring with nursing students, there is minimal known about the specific mentoring behaviors that enhance student achievement. Eller, Lev, and Feurer (2014) found through a qualitative survey study that had purposive sampling from 12 universities from three regions of the United States that there existed eight general themes through content analysis on the key components of the

Citation: Sharon Elizabeth Metcalfe (2017). Developing International Clinical Placements: Enhancing Student Awareness. POJ Nurs Prac Res 1(4): 1-7. 
mentoring relationship for students [14]. These eight themes that were beneficial for students with their clinical mentor were (1) open communication and accessibility: (2) goals and challenges were recognized: (3) passion and inspiration: (4) caring personal relationship: (5) mutual respect and trust: (6) exchange of knowledge: (7) independence and collaboration: and (8) role modeling. These findings suggest that clinical placement mentors have a broad spectrum of positive and beneficial behaviors that enhance the development of the nursing student in the clinical environment.

The role of the clinical mentor was examined by a qualitative study that surveyed a group of nurse mentors that's purpose sought to identify the characteristics of the role that were both rewarding, as well as difficult to fulfill. The results of the study showed that mentors found the role rewarding due to the mentee-mentor relationship that developed to help broaden the knowledge of the mentee. The challenges were due to time pressures, lack of remuneration, and having students that were assigned to them that were indifferent to the placement site [15]. Skela-Savic and Kiger (2015) additionally found that nursing managers often failed to assume responsibility for the professional development of clinical nurse mentors [16].

Nursing students found that clinical mentors who utilized clinical experience stories of their own nursing practice were enriching and helped to understand the students' concerns, sensitivities, and priorities [17]. A final study that utilized an integrative systematic review of 137 articles regarding the role of the mentor in assisting new nursing graduates to be prepared for their novice employment as a registered nurse found that preparation of the clinical preceptor, positive relationships with the novice nurse, and adequate clinical exposure were important for the success of the new nurse as they embarked upon employment [18].

Despite the lack of studies regarding the development of international clinical placements with nursing mentors, there is an abundance of studies that have been conducted on nursing students learning with other professional students through interprofessional education [19]. Interprofessional education transpires when students from two or more professions learn through collaborative educational activities. Huffman, Rosenfield, \& Naismith (2009) found that students from all professions enjoy learning from peer mentoring relationships that develop through interprofessional educational experiences in the clinical setting [20].

In conclusion, the role of the clinical nurse mentor is a vitally important role for both guiding and assisting the nursing student as well as the novice nurse. The literature supports the role of the clinical nurse mentor as a key component to facilitate the learning and knowledge of the student and novice nurse in a wide variety of nursing clinical and educational placements around the globe.

\section{Developing an Initiation Process for Collaborative Exchange}

In 2005, the School of Nursing which is located in the Southeastern United States, sought to expand the diversity of student learning experiences through initiating cultural global exchanges. The purpose of these cultural exchanges would be to facilitate and introduce undergraduate and graduate nursing students to both differences, as well as similarities in patients and delivery of nursing care in developing, as well as developed countries. The purpose of these potential nursing cultural exchanges were discussed with the dean of the international studies program, the dean of the college of health and human sciences, as well as the director of the school of nursing. As the initiation of global travel would be a novel learning experience for students at the university, the three leaders met to discuss the potential growth potential and exposure to both multicultural, as well as ethnic people and places. The university leaders approved the proposal for faculty to initiate cultural exchanges based upon the potential development and merit for students partaking in the learning experiences.

\section{University and Clinical Leadership Contact for University and Clinical Approval}

During the spring of 2006, one of the nursing professors that taught nurses that were returning to school to receive their Bachelors of Science in Nursing, wrote a formal letter to both the university, as well as children's hospital in a district in Great Britain. The purpose of the letter was to seek approval for an onsite visit with a team of professors from the school of nursing in the United States to travel to both the overseas university school of nursing and the children's hospital. The purpose was to discuss the potential for the initiation of a cultural learning exchange program which would include nursing students, nursing professors, and clinical educators from both countries. Nursing directors' from both the university and children's hospital in Great Britain responded positively, and were genuinely interested in pursuing a program of this educational nature. A travel plan for a site visit was developed for the fall of 2006 which would include two professors from the school of nursing in the United States and would be presented to both the director of the school of nursing as well as the dean of the college of health and human sciences.

\section{International Contract for Development of International Clinical Placement Program}

After presentation of the travel plan to the director of the school of nursing and the dean of the college of health and human sciences for initiating the site visit to both the school of nursing and the children's hospital in Great Britain, approval was granted

Citation: Sharon Elizabeth Metcalfe (2017). Developing International Clinical Placements: Enhancing Student Awareness. POJ Nurs Prac Res 1(4): 1-7. 
for travel for the two professors to plan the excursion. The travel to Great Britain was approved to transpire in October 2006, and arrangements for lodging and overseas flight and ground travel were arranged. The travel was arranged to coincide with the week of fall break at the university and school of nursing, and this allowed the professors to conduct the site visit for the potential future exchange trips with undergraduate and graduate students.

Upon arriving in the destination city in Great Britain, the professors were met by representatives from the university and children's hospital to help facilitate the ground travel to the hotel lodging. Arrangements were made to conduct both university and site visits on subsequent days during the week's stay in Great Britain. The professors also arranged to have an opportunity to conduct a meeting with nursing and clinical leaders to discuss in totality the potential plan for both the United States students as well as students from Great Britain to partake in clinical role mentoring placements in the children's hospitals.

During the following days, structured meetings were scheduled with designated representatives of the school of nursing and children's hospital in Great Britain to assess the feasibility of developing a joint exchange program. The program would be based upon nursing students being guided and led through observational clinical placements in both of the children's hospitals that were located in Great Britain, as well as the hospital that was located in the United States. Additionally, the professors of both universities sought to enrich the students' learning experiences through having the students provide presentations that were based on health care issues and concerns that were prevalent in the home country. These presentations would be developed in the home country, and then presented in the visiting country at the school of nursing to nurses, nursing students, nursing professors, and other health care professionals.

Both groups of nursing professors, as well as clinical leaders were found to be in support of developing this unique type of cultural exchange program that would be based upon both student clinical mentoring through observational placements, as well as delivery of student special presentations on health issues of the home country. At the conclusion of the week's meetings, the dean of the school of nursing and the director of nursing of the children's hospital in Great Britain were approached and presented with the cultural exchange plan. Both directors provided their support for the educational learning experience and developed a learning contract that would be signed and sealed through nursing leadership of both universities, the deans of the colleges of health and human sciences, as well as the nursing leaders of both children's hospitals in both countries.

Upon returning to the United States, the visiting team of professors met with both the director of nursing of the school of nursing, the children's hospital and the dean of the college of health and human sciences. The international learning contract was signed by each of the individuals and a copy was sent to the nursing directors of the school of nursing and children's hospital in Great Britain. Arrangements were made to plan the first week of student travel for the United States students to Great Britain during the following spring break. Utilizing a full nine days of the spring break week allowed both nursing undergraduate and graduate students to be able to partake of the rich learning experience without interfering with other courses during the spring break. This tradition of having the travel exchange course being placed during spring break of each year has proven to be successful for both the students as well as the professors in the school of nursing in the United States. For the nursing students that travel to the United States to partake of the learning placements through clinical mentoring at the children's hospital, the students travel and are placed in clinical arrangements during the first two weeks of January each year. These dates coincide with the learning break that the students in Great Britain have and provide a block of time that these students can both observe in clinical placements in the children's hospital, as well as conduct presentations in both the hospital leadership meetings and the school of nursing classes.

\section{Enhancing Student Awareness through Clinical Nursing Mentoring}

The uniqueness of this cultural nursing learning experience is highlighted by the instructional methodology that has been developed for the program. Participating students are asked to submit their request for securing a clinical placement site, as well as a clinical mentor for their observational experiences in the children's hospitals. The professors at both schools of nursing provide this list of preferred observational sites to the two clinical educators at both children's hospitals. The clinical educators request nursing practitioners to carefully guide the student through the preferred site for observational learning. By using observational learning methodology, the student is able to ask pertinent questions of the mentor regarding the care of the child or children in the designated clinical site. This allows each student to receive preferential instruction for specialized units that include the operating room (operating room theater), the emergency room (accident and emergency), critical care (high intensive units), behavioral health (mental health), medical surgical wards, general pediatric care, and specialized units such as neurotrauma and spinal care. Students' observational experiences range from one week for the students traveling from the United States and for two weeks for the students traveling from Great Britain.

Students' presentations from both countries are developed during the semester in the home country prior to the travel overseas. Presentations rangefromawidevariety ofillnessessuch as childhood obesity, childhood behavioral health issues such as autism, childhood diabetes, childhood oncological conditions, and common illnesses such as asthma and allergies. Students' take pride in their presentations and delivery of these are well received in both countries by a diverse group of students as well

Citation: Sharon Elizabeth Metcalfe (2017). Developing International Clinical Placements: Enhancing Student Awareness. POJ Nurs Prac Res 1(4): 1-7. 
as health professionals. Students are thrilled to have delivered their first international presentation to an international audience that consists of various health care providers, as well as nursing professors and academic nurse leaders. The presentations are delivered over a time of 15 minutes and each group of students are allowed time for questions regarding their presentations from the audience. This is one of the highlights of the exchange course and trip for the students traveling to Great Britain and for those students traveling to the United States.

\section{Maturation and Growth through Student Clinical Placements}

The students that partake of the clinical placements and cultural exchange experience develop exponentially in growth and maturation as they learn about the nursing and health care profession with children's care in another country. Another major aspect of their learning is to understand the differences and similarities in the care of children through both a socialized and capitalistic forms of healthcare found in each of the respected countries. Students begin their travel experience with a one day presentation that focuses upon the aspects of flights, ground transportation, clothing, customs of food and meals, respect for nursing and health care professionals in another country, as well as managing money. Additionally, topics of sightseeing and sleeping arrangements are discussed and students are assigned roommates for the duration of the trip. Room arrangements are generally made for students based upon the similarities in age and program of study. For instance, undergraduate students are placed in rooms with similar aged undergraduate students, and graduate students are placed in rooms based upon similar age. Over the eleven years of conducting this program, the professors have found this to be a satisfying experience for the students, and students learn about the lives of their roommate on the trip.

Normally, the students that partake on the clinical excursion do not know one another at the start of the excursion. Throughout the travel experience, the students of both countries have the opportunity to meet with nursing students from the home university of the country that they are visiting. Students are able to meet for dinner in a nearby pub and exchange conversation about their lives and educational processes in their home universities. Students from the United States are always amazed that students from Great Britain specialize as an undergraduate student for a field of study, such as Children's Nurse. Additionally, they are often surprised that nursing students in Great Britain do not take national counsel boards or examinations, such as students do in the United States. This is also a surprise for the students from Great Britain.

\section{Successes and Challenges in the Development of the International Clinical Placements}

Over the years of the international collaborative clinical placement program, there have been many successes and some challenges that have developed with the establishment of a program of this nature. Challenges that have arisen for this program have been lessons learned in the beginning of the program, in which the professors made arrangements for each student for each of the flights, lodging, ground transportation, as well as sightseeing excursions during the course for the students. This quickly became a difficult situation, as the professors were actively collecting a large sum of money from each student and placing this money in the universities financial department for safe keeping while arrangements were made for flights, ground travel, and tourist opportunities. The professors that developed the clinical placement program rapidly deduced that this was a difficult situation to be responsible for a sizeable amount of money while teaching simultaneously in university courses and clinical laboratories.

During the second year of the program, the lead professor of the course was introduced to a commercial university educational travel company that focused upon well designed courses and included the collection of all funds that were necessary for flights, lodging, ground travel, as well as a variety of tourist travel opportunities. From this second year of the program onward, the university in the United States has utilized the custom services of the commercial university educational travel company. The advantages of this arrangement have also included a specialized trip coordinator that was assigned to work with the lead professor of the trip and to clarify any questions or concerns that arose prior to the trip, as well as after the trip's conclusion. This arrangement has been a successful change to a potentially major challenge for a trip of this nature that involved a large number of students and a sizeable amount of funds.

Successful outcomes for this program have been many throughout the years of the program. The growth in awareness of each student as they have become more astute in their learning regarding the nature of the nursing profession in the delivery of pediatric care delivered in both countries has been phenomenal. Students become sources of knowledge regarding the profession of nursing and the differences and similarities in caring for children in both countries. They are then able to express this through presentations that they make to their classmates upon return in their home university environments. For many students, they return to their home country a changed nursing student that has developed a new professional awareness and perspective of a culture and delivery of nursing care that is uniquely different from their own home country. Students return to their home country with a very special appreciation of the leadership role of the clinical placement mentor that spent a week with them both guiding and directing them through observational placements of the care of children. For many students, this is the first time that they have been formalized as to the role of a clinical nursing specialist, and return home with a better understanding to share with their peers.

Citation: Sharon Elizabeth Metcalfe (2017). Developing International Clinical Placements: Enhancing Student Awareness. POJ Nurs Prac Res 1(4): 1-7. 


\section{Recommendations and Suggestions for Nurse Faculty and Clinical Leaders}

Throughout the past decade of delivery of the clinical placement program that has involved two universities and two children's hospitals within the United States and Great Britain, there are several major recommendations that can be offered to university faculty and hospital clinical leaders that are interested in developing a similar program. The following list provides an insight to recommendations that may be helpful to others that seek to establish a similar program:

1) For each course/trip in which students are introduced to traveling overseas, plan to introduce the course a year in advance. This will allow students to save money for the excursion and to be able to pay for the costs of the trip.

2) Utilize the services of a professional travel coordinator that works exclusively with an educational travel company that will be able to guide the professors in the many details that need to be arranged for a trip of this size and nature to a foreign country.

3) Develop an international learning contract that allows all parties (universities, as well as hospital leaders) to thoroughly appraise themselves of the contents of the learning contract and have time for attorneys to review the details of the contract.

4) Allow adequate time to have college leadership and hospital leadership sign the international learning contracts and submit to their attorneys for safe keeping.

5) Develop an international travel course that is based upon observational clinical placement opportunities with a nursing mentor one year prior to trip. In many universities, this process takes up to one year to develop a course and seek approval for granting of credit for students that sign up to take the course. For the majority of students, students will desire to receive elective credit hours for the course.

6) And finally, introduce to students the concept that they will be embarking on an excursion to a country that they have never been to, and approach the culture, the food, the people, the hospital settings, and the university environments, in a positive and welcoming approach. This is truly a once-in-a-lifetime opportunity!

Educational travel excursions that are developed through a joint collaboration with both university nursing leadership and hospital leadership from both home countries can be a fulfilling endeavor for students, faculty, and clinical nursing specialists, leadership and educators. It is hoped that the recommendations suggested in this article will provide guidance and leadership for other professors and clinical leaders that wish to embark on a journey such as this.

\section{References}

1. Berland A, Richards J, Lund KD. A Canada-Bangladesh partnership for nurse education: Case study. Int Nurs Rev. 2010;57(3):352-358.

2. Missing Persons: Minorities in the Health Professions, A Report of the Sullivan Commission on Diversity in the Healthcare Workforce. Durham, NC: Sullivan Commission; 2004.

3. Institute of Medicine. The Future of Nursing Report: Leading change, advancing health. Institute of Nursing, The Robert Wood Johnson Foundation. Washington (DC): National Academies Press (US); 2010.

4. Gilchrist KL, Rector C. Can you keep them? Strategies to attract and retain nursing students from diverse populations: Best practices in nursing education. J Transcult Nurs. 2007;18(3):277-285.

5. Budden JS, Zhong EH, Moulton P, Cimiotti JP. Highlights of the national workforce survey of registered nurses. J Nurs Regul. 2013;4(2):5-14.

6. The United States Census Bureau. Statistical Abstract of the United States: 2012. Washington (DC): U.S. Department of Commerce; 2012.

7. Jie $\mathrm{H}$, Andreatta S, Liping Y, Sijian L. A collaborative international community health nursing: Clinical experiences in China. Home Health Care Manag Pract. 2010;22(7):499-506.

8. Smith K, Curry K. Is it worth it? Measuring the long-term effects of an international experience for nursing students in Ecuador. J Community Health Nurs. 2011;28(1):14-22.

9. Kolbuck PA, Mitchell EM, Glick DF, Griener D. International experiences in nursing education: $A$ review of the literature. Int J Nurs Educ Scholarsh. 2012;9:1-21.

10. Dobrowolska B, McGonagle I, Kane R, Jackson CS, Kegl $B$, Bergin $M$, et al. Patterns of clinical mentorship in undergraduate nurse education: A comparative case analysis of eleven EU and non-EU countries. Nurse Educ Today. 2016;36:44-52.

11. Ward A, McComb S. Precepting: A literature review. J Prof Nurs. 2017;33(5):314-325.

12. Forber J, DiGiacomo M, Carter B, Davidson P, Phillips J, Jackson D. In pursuit of an optimal model of undergraduate nurse clinical education: An integrative review. Nurse Educ Pract. 2016;21:83-92.

Citation: Sharon Elizabeth Metcalfe (2017). Developing International Clinical Placements: Enhancing Student Awareness. POJ Nurs Prac Res 1(4): 1-7. 
13. Roxburgh M. Undergraduate student nurses' perceptions of two practice learning models: A focus group study. Nurse Educ Today. 2014;34(1):40-46.

14. Eller LS, Lev EL, Feurer A. Key components of an effective mentoring relationship: A qualitative study. Nurse Educ Today. 2014;34(5):815-820.

15. Rylance R, Barrett J, Sixsmith P, Ward D. Student nurse mentoring: An evaluative study of the mentor's perspective. Br J Nurs. 2017;26(7):405-409.

16. Skela-Savic B, Kiger A. Self-assessment of clinical nurse mentors as dimensions of professional development and the capability of developing ethical values at nursing students: A correlational research study. Nurse Educ Today. 2015;35(10):1044-1051.
17. Edwards S. What nursing students reveal about and learn from mentors when using stories of clinical practice. Nurs Manag (Harrow). 2017;23(10):32-39.

18. Edward KL, Ousey K, Playle J, Giandinoto JA. Are new nurses work ready- The impact of preceptorship. An integrative systematic review. J Prof Nurs. 2017;33(5):326333.

19. Buring SM, Bhushan A, Broeseker A, Conway S, DuncanHewitt W, Hansen $L$, et al. Interprofessional education: Definitions, student competencies, and guidelines for implementation. Am J Pharm Educ. 2009;73(4):59.

20. Hoffman SJ, Rosenfield D, Nasmith L. What attracts students to interprofessional education and other health care reform initiatives? J Allied Health. 2009;38(3):75-78.

Citation: Sharon Elizabeth Metcalfe (2017). Developing International Clinical Placements: Enhancing Student Awareness. POJ Nurs Prac Res 1(4): 1-7. 